\title{
Significant Long-term Variations in Left Ventricular Ejection Fraction Driven by Systemic Vascular Resistance Changes
}

\section{Damien MANDRY}

Université de Lorraine

Nicolas GIRERD

Université de Lorraine

Zohra LAMIRAL

Centre Hospitalier Universitaire de Nancy

Olivier HUTTIN

Centre Hospitalier Universitaire de Nancy

\section{Laura FILIPPETTI}

Centre Hospitalier Universitaire de Nancy

Micard Emilien

Inserm

Marine BEAUMONT

Inserm

Marie-Paule Bernadette NCHO MOTTOH

Centre Hospitalier Universitaire de Nancy

Nathalie PACE

Centre Hospitalier Universitaire de Nancy

Faïez ZANNAD

Université de Lorraine

Patrick ROSSIGNOL

Université de Lorraine

Pierre-Yves MARIE ( $\nabla$ py.marie@chru-nancy.fr)

Université de Lorraine

\section{Research Article}

Keywords: Cardiac MRI, flow-encoding sequence, ejection fraction, systemic vascular resistance, hypertension, obesity

Posted Date: June 18th, 2021 
DOI: https://doi.org/10.21203/rs.3.rs-617555/v1

License: (c) (1) This work is licensed under a Creative Commons Attribution 4.0 International License. Read Full License 


\section{Abstract}

This study aims to determine whether changes in vascular function parameters, such as systemic vascular resistance (SVR), might explain the significant changes in LV ejection fraction (EF) that may be observed over the long term in subjects with no evident health issues.

Subjects with no evident health issues except for hypertension and obesity, underwent MRI with phasecontrast sequences at baseline and at a median follow-up of 5.2 years. Longitudinal changes in EF were analyzed with regard to concomitant changes in the vascular function parameters computed with an MRI flow-sequence.

A total of 118 subjects ( $53 \pm 12$ years) were included among whom, $52 \%$ were women, $26 \%$ had hypertension, and $52 \%$ were obese. The longitudinal change in EF was inversely related to concomitant changes in mean and diastolic blood pressure $(p=0.030$ and $p=0.027$, respectively) and much more significantly so, in SVR $(p<0.001)$. SVR changes were $-8.08 \pm 9.21$ and $+8.14 \pm 8.28 \mathrm{mmHg} \cdot \mathrm{min} \cdot \mathrm{m}^{2} \cdot \mathrm{L}^{-1}$ respectively in subjects with significant increases $(n=7)$ and decreases $(n=10)$ in $E F$, and $3.32 \pm 7.53$ mmHg.min $\cdot m^{2} \cdot L^{-1}$ in subjects with stable EF $(n=100)$ (overall $\left.p<0.001\right)$.

The EF variations of populations with no evident health issues, except for hypertension and obesity, are mostly driven by concomitant changes in arterial function rather than in LV contractility.

Clinical Trial Registrations. NCT01716819 and NCT02430805 on the ClinicalTrials.gov site.

\section{Introduction}

The left ventricular (LV) ejection fraction (EF) remains extensively used to quantify LV systolic performance ${ }^{1}$, and MRI is a reference technique for measuring EF and monitoring EF changes ${ }^{1,2-4}$. However, irrespective of the imaging technique used for measuring the $E F$, the main limitation is its dependence upon loading conditions, analogously to most other parameters used to assess LV systolic function ${ }^{5}$. The EF is not constant in any subject but varies, particularly as a function of the afterload and blood pressure $(\mathrm{BP})^{6-8}$. Thus, it is commonly recommended to report the $\mathrm{BP}$ level observed during any EF measurement ${ }^{9-11}$.

At the present time, however, LV afterload may be assessed in a more precise but still non-invasive way by combining the information from brachial BP to the stroke volume values provided by MRI flow-velocity sequences ${ }^{12-14}$. This approach allows to measure several parameters known to reflect or to greatly impact the LV afterload, notably systemic vascular resistance (SVR), effective arterial elastance (Ea), and total arterial compliance (TAC) $)^{12-14}$.

In a previous MRI study, the evolution of EF and SVR over time were shown to be interdependent in the months following an acute myocardial infarction (MI), with the increase in EF being linked to the concomitant decrease in SVR under the post-MI vasodilating medical regimen ${ }^{13}$. However, it is not known 
whether such SVR changes might also explain the significant EF variations which may be observed in subjects with no evident cardiac disease but at increased cardiovascular risk, such as hypertensive and/or obese subjects.

The present MRI-based longitudinal study aims to determine whether longitudinal changes in LV afterload, and particularly in SVR, might explain the significant changes in EF observed over the long term in subjects with no evident health issues except for uncomplicated hypertension and obesity.

\section{Materials And Methods}

\section{Study Populations}

Subjects in the current study did not have any medical history of cardiac disease. They underwent cardiovascular monitoring with the same MRI protocol and were pooled from two different cohorts:

1) The "Role of the Renin Angiotensin Aldosterone System in the Mechanisms of Transition to Heart Failure in Abdominal Obesity (R2C2-II)" cohort has already been described elsewhere ${ }^{12,14}$. This cohort includes middle-aged subjects ( 40 to 65 years) with an abdominal obesity, with no cardiac disease and no cardiovascular medication, except for a stage 1 hypertension. Subjects were invited to participate in a $>4$-year longitudinal study, which included MRI at baseline and another one at follow-up (ClinicalTrials.gov NCT01716819).

2) The "Predisposition and Transition Mechanisms from Arterial Hypertension to Heart Failure (Hypercare)" family-based study included 58 subjects aged below 60, with uncomplicated hypertension and a history of familial hypertension. This longitudinal study, which included MRI investigations at baseline, and at 2- and 4-year intervals, has already been published elsewhere ${ }^{12}$ (ClinicalTrials.gov NCT02430805).

The main exclusion criteria, which were common to the two cohorts, were: diabetes; inflammatory or infectious disease; renal, hepatic or pulmonary insufficiency; and history of malignant diseases. Both cohort-studies were approved by the local Ethics Committee, with all study participants providing their signed informed consent to participate. All methods were performed in accordance with the relevant guidelines and regulations.

\section{MRI recording and analysis}

MRIs were performed on a 3-T or 1.5-T magnet (GE Medical Systems, Milwaukee, WI, USA) ${ }^{12}$. An automated sphygmomanometer (Maglife C, Schiller Medical, Wissembourg, France) was used to measure brachial blood pressure (BP) during the MRI examinations. Averaged values were used for the analyses presented below.

A steady-state free precession pulse sequence and a dedicated software (MASS ${ }^{\mathrm{TM}}$, Medis, The Netherlands) were used to measure LV end-diastolic volume, end-diastolic mass, and EF in contiguous 
short-axis, and the concentric remodeling (CR) index was defined as LV mass / end-diastolic volume ratio $^{12-14}$.

Cardiac flow was determined using a velocity-encoded phase-contrast gradient-echo sequence on a slice positioned perpendicularly to the ascending aorta ${ }^{12-14}$. The stroke volume (SV) was obtained with the "CV flow" software (Medis, The Netherlands), with velocities corrected using an ROI-based method only in instances of obvious offset error.

The values of cardiac flow and stroke volume were used to estimate the systemic vascular resistances (SVR: mean pressure/cardiac flow) $)^{12-18}$, effective arterial elastance $(($ Ea $=0.9 \times$ systolic BP $(\mathrm{mmHg}) /$ stroke volume $(\mathrm{mL})^{12,15-18}$, and total arterial compliance index $(\mathrm{TAC}=$ stroke volume $(\mathrm{mmHg}) /$ pulse pressure $(\mathrm{mmHg}))^{12,14-16}$. All these cardiovascular MRI-derived parameters were indexed to body surface area, except for $\mathrm{EF}$ and $\mathrm{CR}$.

As already detailed elsewhere, abnormally high values of SVR, LV mass and concentric remodeling index were defined as the upper limits of the $95 \%$ confidence intervals observed in a healthy non-obese middleage population investigated with the same MRI methodology ${ }^{12}$. Significant EF change was additionally defined as an absolute difference $>8 \%$ according to a reproducibility study also performed with the same MRI methodology in our center ${ }^{13}$.

\section{Statistical analyses}

Analyses were performed using the commercially available SAS software version 9.4 (SAS Institute Inc. Cary, NC, USA). Continuous variables are expressed as mean and standard deviations (SD) and categorical variables as numbers and percentages (Table 1). Paired comparisons between baseline and follow-up were evaluated using the Wilcoxon sum rank test for continuous variables and the Mc Nemar test for categorical variables. Spearman correlation coefficients and their $95 \% \mathrm{Cl}$ intervals were computed for the baseline-to-follow-up changes in EF and the selected variables listed in Table 2. Univariate and multivariate ascending regression analyses were performed while checking Linear model assumptions and with a $p$ value $<0.05$ to enter variables and $>0.10$ to remove variables (Table 3 ). 
Table 1

Comparison of the main recorded data between baseline and follow-up

\begin{tabular}{|c|c|c|c|}
\hline & Baseline & Follow-up & $P$ value \\
\hline Age (years) & $52.5 \pm 12.5$ & $58.5 \pm 12.3$ & \\
\hline Female gender & $61(51.7 \%)$ & $61(51.7 \%)$ & \\
\hline Body weight (kg) & $83.8 \pm 14.7$ & $86.5 \pm 15.0$ & 0.0004 \\
\hline Body mass index (kg.m-2) & $29.6 \pm 4.8$ & $30.7 \pm 4.9$ & $<0.0001$ \\
\hline Obesity & $61(51.7 \%)$ & $65(55.1 \%)$ & 0.80 \\
\hline Body mass index (kg.m $\left.{ }^{-2}\right)$ & $29.6 \pm 4.8$ & $30.7 \pm 4.9$ & $<0.0001$ \\
\hline Heart rate (bpm) & $70.3 \pm 11.5$ & $65.9 \pm 10.7$ & 0.002 \\
\hline Systolic BP (mmHg) & $128.7 \pm 18.1$ & $128.7 \pm 16.3$ & 0.60 \\
\hline Diastolic BP (mmHg) & $74.7 \pm 12.3$ & $76.3 \pm 10.4$ & 0.045 \\
\hline Mean BP (mmHg) & $92.4 \pm 13.2$ & $93.4 \pm 10.8$ & 0.15 \\
\hline Pulse BP (mmHg) & $54.0 \pm 12.9$ & $52.4 \pm 13.4$ & 0.13 \\
\hline Indexed stroke volume $\left(\mathrm{mL} . \mathrm{m}^{-2}\right)$ & $43.1 \pm 8.9$ & $41.9 \pm 8.8$ & 0.44 \\
\hline Cardiac index $\left(\right.$ L. $\left.\mathrm{min}^{-1} \cdot \mathrm{m}^{-2}\right)$ & $3.00 \pm 0.66$ & $2.72 \pm 0.53$ & $<0.0001$ \\
\hline Indexed SVR (mmHg.min $\left.\cdot \mathrm{m}^{2} \cdot \mathrm{L}^{-1}\right)$ & $32.1 \pm 7.9$ & $35.6 \pm 7.6$ & $<0.0001$ \\
\hline abnormal (> 40 mmHg.min.m ${ }^{2} \cdot L^{-1}$ ) & $17(14.4 \%)$ & $29(24.6 \%)$ & 0.45 \\
\hline Indexed TAC (mL.mmHg$\left.{ }^{-1} \cdot \mathrm{m}^{-2}\right)$ & $0.84 \pm 0.23$ & $0.85 \pm 0.27$ & 0.65 \\
\hline Indexed Ea (mmHg.mL $\left.{ }^{-1} \cdot \mathrm{m}^{2}\right)$ & $2.79 \pm 0.67$ & $2.89 \pm 0.76$ & 0.40 \\
\hline Indexed ESV (mL.m² $)$ & $28.9 \pm 7.5$ & $28.3 \pm 7.6$ & 0.15 \\
\hline Indexed EDV (mL.m²) & $71.8 \pm 12.2$ & $69.3 \pm 13.0$ & 0.006 \\
\hline $\mathrm{EF}(\%)$ & $60.0 \pm 6.3$ & $59.6 \pm 5.3$ & 0.46 \\
\hline abnormal $(<50 \%)$ & $5(4.2 \%)$ & $4(3.4 \%)$ & 0.56 \\
\hline Indexed LV mass $\left(\mathrm{g} \cdot \mathrm{m}^{-2}\right.$ ) & $50.5 \pm 10.5$ & $48.2 \pm 9.6$ & $<0.0001$ \\
\hline CR index $\left(\mathrm{g} \cdot \mathrm{mL}^{-1}\right)$ & $0.71 \pm 0.15$ & $0.71 \pm 0.14$ & 0.19 \\
\hline \multicolumn{4}{|c|}{$\begin{array}{l}\mathrm{BP}=\text { blood pressure; } \mathrm{CR}=\text { concentric remodeling; } \mathrm{Ea}=\text { effective arterial elastance; } E D V=\text { end-diastolic } \\
\text { volume; } \mathrm{EF}=\text { ejection fraction; } \mathrm{LV}=\text { left ventricle; } \mathrm{TAC}=\text { total arterial compliance; } \mathrm{SVR}=\text { systemic } \\
\text { vascular resistances. }\end{array}$} \\
\hline
\end{tabular}


Table 2

Association between longitudinal changes in LVEF and baseline and concomitant changes in clinical and hemodynamic variable

\begin{tabular}{|c|c|c|}
\hline Parameter & $r_{s}(95 \% \mathrm{Cl})$ & P-value \\
\hline Female gender & $-0.60(-2.05 .0 .84)$ & 0.72 \\
\hline Body mass index $\left(\mathrm{kg} \cdot \mathrm{m}^{-2}\right)$ & $-0.01(-0.19,0.17)$ & 0.91 \\
\hline$\Delta$ from baseline & $-0.09(-0.27,0.09)$ & 0.31 \\
\hline Age (years) & $-0.13(-0.30,0.05)$ & 0.16 \\
\hline$\Delta$ from baseline & $0.02(-0.16,0.20)$ & 0.84 \\
\hline Heart rate (bpm) & $0.02(-0.16,0.20)$ & 0.84 \\
\hline$\Delta$ from baseline & $-0.10(-0.28,0.08)$ & 0.27 \\
\hline Systolic BP (mmHg) & $-0.03(-0.21,0.16)$ & 0.78 \\
\hline$\Delta$ from baseline & $-0.08(-0.26,0.10)$ & 0.38 \\
\hline Diastolic BP (mmHg) & $0.06(-0.12,0.24)$ & 0.52 \\
\hline$\Delta$ from baseline & $-0.20(-0.37,-0.02)$ & $0.027 *$ \\
\hline Mean BP (mmHg) & $0.06(-0.12,0.24)$ & 0.50 \\
\hline$\Delta$ from baseline & $-0.20(-0.36,-0.02)$ & $0.031^{*}$ \\
\hline Pulse BP (mmHg) & $-0.10(-0.27,0.09)$ & 0.30 \\
\hline$\Delta$ from baseline & $0.10(-0.08,0.27)$ & 0.29 \\
\hline Baseline EF & $-0.63(-0.73,-0.50)$ & $<0.0001$ \\
\hline Indexed SVR (mmHg.min. $\left.\mathrm{m}^{2} \cdot \mathrm{L}^{-1}\right)$ & $0.32(0.14,0.47)$ & $0.0005^{\star}$ \\
\hline$\Delta$ from baseline & $-0.44(-0.57,-0.28)$ & $<0.0001$ \\
\hline Indexed TAC (mL.mmHg$\left.{ }^{-1} \cdot \mathrm{m}^{-2}\right)$ & $-0.13(-0.30,0.06)$ & 0.18 \\
\hline$\Delta$ from baseline & $0.19(0.01,0.36)$ & $0.038^{\star}$ \\
\hline Indexed Ea $\left(\mathrm{mmHg} \cdot \mathrm{mL}^{-1} \cdot \mathrm{m}^{2}\right)$ & $-0.17(-0.34,0.01)$ & 0.059 \\
\hline$\Delta$ from baseline & $-0.42(-0.56,-0.26)$ & $<0.0001$ \\
\hline
\end{tabular}


Table 3

Linear regression models obtained through forward selections, with Beta coefficients (standard error (SE)), $P$ and $R^{2}$ values, for predicting the follow-up to baseline differences in EF (A). The model was additionally built after exclusion of the baseline EF value (B).

\begin{tabular}{|lllll|}
\hline & & Beta (SE) & P-value & Global R \\
\hline A & Intercept & $30.83 \pm 3.66$ & $<0.0001$ & \\
& Change in SVR & $-0.22 \pm 0.05$ & $<0.0001$ & \\
& Baseline EF & $-0.51 \pm 0.06$ & $<0.0001$ & 0.50 \\
\hline B & Intercept & $0.51 \pm 0.50$ & 0.31 & \\
& Change in SVR & $-0.30 \pm 0.06$ & $<0.0001$ & 0.19 \\
\hline
\end{tabular}

\section{Results}

\section{Baseline characteristics of the study population}

The study population consisted of a total of 118 subjects, 44 from the Hypercare cohort and 74 from the R2C2 cohort. Based on the inclusion criteria, none of these 118 subjects had any history of cardiovascular disease except for an uncomplicated hypertension in 31 participants (26\%). None had any significant cardiac abnormalities by MRI, except for 5 subjects with an EF below the $50 \%$ level (EF ranging from 43 to $47 \%$ ) as well as 3 and 17 subjects with a higher-than-normal LV mass and CR index, respectively.

As detailed in Table 1, mean age was $53 \pm 12$ years, 62 (52\%) were women, and 61 (52\%) were mild to moderately obese with body mass indexes ranging from 30 to $40 \mathrm{~kg} \cdot \mathrm{m}^{-2}$.

\section{Evolution at follow-up}

Follow-up investigations, performed at a median of 5.2 years from baseline (range 3.7 to 8.4 years), provided evidence of significant increases in body weight, body mass index, diastolic BP and SVR, compared to baseline (Table 1). Significant decreases in heart rate, cardiac index, end-diastolic volume and indexed LV mass were additionally evident (Table 1).

Eighteen subjects (15\%) had significant EF variations (> 8\%) between baseline and follow-up, 7 with an increased EF and 11 with a decreased EF. These variations were in line with changes in LV end-systolic volume $(p<0.001)$ but not end-diastolic LV volumes.

Only 4 subjects had $<50 \%$ EF at follow-up. Among the 5 subjects with $<50 \%$ EF at baseline, only one still had $a<50 \%$ EF at follow-up, whereas the 4 others had EF increases which exceeded the $50 \%$ level at follow-up. 


\section{Correlates of baseline-to-follow-up changes in EF}

As detailed in Table 2, the difference in EF between follow-up and baseline was inversely correlated to the corresponding differences in mean BP $(p=0.03)$ and diastolic BP $(p=0.027)$.

However, this predictive value of BP-changes was much lower than those provided by the baseline-tofollow-up changes in effective arterial elastance (Ea) and in SVR (both $p<0.001$, Table 2). The baseline levels of EF, Ea and SVR were additional univariate predictors, whereas all other analyzed parameters were not (Table 2).

On the multivariate regression analysis, which considered all the significant univariate predictors from Table 2, EF changes were significantly and independently related to the baseline-to-follow-up change in SVR together with the baseline EF value (see Table 3). Only the follow-up change in SVR was kept in the model after baseline EF had been excluded (Table 3). Figure 1 displays the inverse association between the follow-up changes in EF and SVR.

As illustrated in Fig. 2, the baseline-to-follow-up changes in SVR were markedly different between subjects with significant increase in EF during follow-up and those with significant decrease in EF (-8.08 $\pm 9.21 \mathrm{mmHg} \cdot \mathrm{min} \cdot \mathrm{m}^{2} \cdot \mathrm{L}^{-1}$ vs. $\left.8.14 \pm 8.28 \mathrm{mmHg} \cdot \mathrm{min} \cdot \mathrm{m}^{2} \cdot \mathrm{L}^{-1}, \mathrm{p}<0.001\right)$. The remaining group of subjects with stable EF had no significant baseline-to-follow-up change in SVR (SVR difference in this group: 3.32 $\left.\pm 7.53 \mathrm{mmHg} \cdot \mathrm{min} \cdot \mathrm{m}^{2} . \mathrm{L}^{-1}\right)$ (Fig. 2).

\section{Discussion}

In a population including hypertensive and obese subjects but none with any history of cardiac disease, the present MRI study shows that significant long-term EF variations are not negligible, affecting some $15 \%$ of this study population. Moreover, most of these variations are likely driven by changes in SVR and thus, not by real changes in LV contractility.

SVR is the main component of the LV afterload, reflecting the opposition of the microcirculation that must be overcome by the LV to eject blood. In the present study population, mean SVR exhibited an increase over time (Table 1), providing evidence of an average trend towards a functional deterioration of the microcirculation. Such a deterioration is common during the aging process, together with an increase in the stiffness of large arteries, and it may be further promoted by hypertension and obesity ${ }^{19,20}$. It is likely that the high rates of hypertension (26\%) and obesity (52\%) in the present study population accelerate the rate of SVR deterioration, consequently impacting the EF.

The interdependence of the EF on cardiac loading conditions is well established, but despite this limitation, EF remains extensively used to quantify LV systolic performance ${ }^{1}$. Because of this loaddependence, the current recommendation is to report brachial BP for each EF measurement ${ }^{9-11}$. The change in BP was a significant predictor of EF change in the present MRI study, but only for diastolic and 
mean BP levels. Diastolic BP has already been shown to have a greater impact on EF than systolic BP, particularly in heart failure with preserved $\mathrm{EF}^{6}$. Diastolic $\mathrm{BP}$ is likely to sustain blood flow through the microvasculature ${ }^{21,22}$ and would thus strongly depend on the resistance of small vessels. This is in contrast to the stiffness of great arteries which would have a greater impact on pulse pressure and systolic $\mathrm{BP}^{23}$.

Nevertheless, more precise functional arterial parameters than brachial BP were collected here with the use of conventional MRI flow sequences. These allow to determine aortic stroke volume independently of the other MRI sequences used to assess LV function and moreover, with mean flow and vessel diameter measurements that are more precise than those determined using Doppler techniques ${ }^{24}$. By combining these stroke volume values with brachial BP measurements, three functional vascular parameters were computed in the present study: (i) the total arterial compliance index (TAC), which is predominantly governed by the elastic great arteries ${ }^{12,14-16}$, (ii) the systemic vascular resistance (SVR), which is mainly attributed to the resistive microvessels ${ }^{12-18}$, and (iii) the global arterial load (Ea), a comprehensive measure of the arterial load that depends on both arterial compliance and arterial resistance ${ }^{12,15-18}$.

Ea changes over time were previously shown to be strongly predictive of the evolution of LV remodeling ${ }^{12}$. In the present study, however, Ea-changes were not found to be better predictors of EF variations than SVR-changes, similarly to what was previously documented in post-myocardial infarction patients ${ }^{13}$. This is presumably because the small arteries governing the SVR not only constitutes the main component of the LV afterload but also, the exit door through which the stroke volume needs to pass before leaving the arterial tree. Therefore, it could be speculated that all changes in SVR would more directly impact stroke volume and EF, than Ea changes do.

A significant decrease in SVR has already been shown to be the main mechanism by which the EF increases during exercise in heart disease patients ${ }^{25}$, and during the months following a myocardial infarction ${ }^{11}$. The observation of a frequent decrease in SVR after infarction, has been attributed to the introduction of a vasodilator medical regimen (i.e., Angiotensin Converting Enzyme Inhibitors or Angiotensin Receptor Blockers) ${ }^{11}$. Such a vasodilator-related decrease in SVR was additionally shown to be associated with proportional increases in the stroke volume and cardiac output in heart failure patients ${ }^{26}$.

It is also worth mentioning that in addition to the SVR changes, the baseline EF level, was an independent predictor of EF variations over time. The impact of baseline EF could at least partly be attributed to a regression to the mean - i.e., a statistic phenomenon that implies that a sample point of a random variable, which is distant from the mean value on a first measurement, has a high probability to be closer to the mean value on a second measurement. This point is better explained and further illustrated in Fig. 3. 
The present study defined a significant EF variation using an absolute threshold of $8 \%$, which corresponds to results of a reproducibility analysis previously reported by our team with the same MRImethodology ${ }^{13}$. However, it has been shown that this threshold can vary, depending on the different conditions tested in the reproducibility analysis (inter- or intra-observer measurements, single or different time-points, single or different scanners ${ }^{27}$ ). Nevertheless, this does not modify the consideration that the rate of EF changes over time may be significantly lowered after adjusting for the concomitant SVRchanges observed with MRI. Such an adjustment would help identify situations where a decrease in EF relates to vascular rather than myocardial deteriorations and thus, where the therapeutic target is not the same.

\section{Conclusions}

The present longitudinal MRI study of a cohort including hypertensive and obese subjects shows that significant long-term variations in $\mathrm{EF}$ are not uncommon, but most of these variations are likely driven by changes in SVR rather than in LV contractility. This underscores the usefulness of additional SVR measurements when EF is monitored by MRI in populations at risk of vascular dysfunction.

\section{Abbreviations}

BP

blood pressure

$\mathrm{CR}$

concentric remodeling

Ea

effective arterial elastance

$\mathrm{EF}$

ejection fraction

LV

left ventricle

$\mathrm{MI}$

myocardial infarction

MRI

magnetic resonance imaging

SV

stroke volume

SVR

systemic vascular resistance

TAC

total arterial compliance 


\section{Declarations}

\section{ACKNOWLEDGEMENTS}

The authors wish to thank Petra Neufing for critical review of the manuscript.

Data Availability: The datasets used and/or analyzed during the current study are available from the corresponding author on reasonable request.

Ethics approval and consent to participate: The 2 cohort-studies involved in the present article were approved by the local Ethics Committee (Comité de Protection des Personnes se prétant à la recherche biomédical (CPP) Nancy), with all study participants providing their signed informed consent to participate.

Competing interests: The authors disclose no relevant competing interests relevant to this research.

FUNDING: National Health Ministry (Programme Hospitalier de Recherche Clinique), the $6^{\text {th }}$ framework program of the European Commission (Ingenious HyperCare Network of Excellence; contract number LSHM-CT-2006-037093).

Contributions: Author contributions are as follows: conception and study design (NG, FZ, PR, PYM), data collection and protocol design (DM, OH, LP, EM, MB, MBNM, NP), data analysis and interpretation (DM, $\mathrm{ZL}, \mathrm{OH}, \mathrm{FZ}, \mathrm{PYM}$ ), drafting (DM, OH, FZ, PYM) and revising (DM, NG, ZL, OH, FZ, PR, PYM) of the paper. All authors read and approved the final manuscript.

\section{References}

1 - Harbo MB, Nordén ES, Narula J, et al. Quantifying left ventricular function in heart failure: What makes a clinically valuable parameter? Prog Cardiovasc Dis. 2020;63(5):552-60.

2 - Mavrogeni S, Katsi V, Vartela V, et al. The emerging role of Cardiovascular Magnetic Resonance in the evaluation of hypertensive heart disease. BMC Cardiovasc Disord. 2017; 17(1):132.

3 - Moody WE, Edwards NC, Chue CD, et al. Variability in cardiac MR measurement of left ventricular ejection fraction, volumes and mass in healthy adults: defining a significant change at 1 year. $\mathrm{Br} \mathrm{J}$ Radiol. 2015;88(1049):20140831.

4 - Fratz S, Chung T, Greil GF, et al. Guidelines and protocols for cardiovascular magnetic resonance in children and adults with congenital heart disease: SCMR expert consensus group on congenital heart disease. J Cardiovasc Magn Reson. 2013; 15: 51.

5 - Dahl JS, Carter-Storch R. First-Phase Ejection Fraction: The FEV1 of the Heart? JACC Cardiovasc Imaging. 2019;12(1):64-66. 
6 - Wei FF, Xue R, Thijs L, et al. Associations of Left Ventricular Structure and Function With Blood Pressure in Heart Failure With Preserved Ejection Fraction: Analysis of the TOPCAT Trial. J Am Heart Assoc. 2020; 9(15):e016009.

7 - Wolz DE, Flores AR, Grandis DJ, et al. Abnormal left ventricular ejection fraction response to mental stress and exercise in cardiomyopathy. J Nucl Cardiol. 1995;2(2 Pt 1):144-50.

8 - Bairey CN, de Yang L, Berman DS, et al. Comparison of physiologic ejection fraction responses to activities of daily living: implications for clinical testing. J Am Coll Cardiol. 1990;16:847-54.

9 - Galderisi M, Cosyns B, Edvardsen T, et al. Standardization of adult transthoracic echocardiography reporting in agreement with recent chamber quantification, diastolic function, and heart valve disease recommendations: an expert consensus document of the European Association of Cardiovascular Imaging. Eur Heart J Cardiovasc Imaging. 2017;18(12):1301-10.

10 - Hesse B, Lindhardt TB, Acampa W, et al. EANM/ESC guidelines for radionuclide imaging of cardiac function. Eur J Nucl Med Mol Imaging. 2008;35(4):851-85.

11 - Galderisi M, Henein MY, D'hooge J, et al. Recommendations of the European Association of Echocardiography: how to use echo-Doppler in clinical trials: different modalities for different purposes. Eur J Echocardiogr. 2011;12(5):339-53.

12 - Marie PY, Mandry D, Huttin O, et al. Comprehensive monitoring of cardiac remodeling with aortic stroke volume values provided by a phase-contrast MRI sequence. J Hypertens. 2016; 34(5):967-73.

13 - Huttin O, Mandry D, Eschalier R, et al. Cardiac remodeling following reperfused acute myocardial infarction is linked to the concomitant evolution of vascular function as assessed by cardiovascular magnetic resonance. J Cardiovasc Magn Reson. 2017; 19(1):2.

14 - Mandry D, Eschalier R, Kearney-Schwartz A, et al. Comprehensive MRI analysis of early cardiac and vascular remodeling in middle-aged patients with abdominal obesity. J Hypertens. 2012; 30(3):567-73.

15 - Otsuki T, Maeda S, lemitsu M, et al. Contribution of systemic arterial compliance and systemic vascular resistance to effective arterial elastance changes during exercise in humans. Acta Physiol (Oxf). 2006; 188(1):15-20.

16 - Otsuki T, Maeda S, lemitsu M, et al. Systemic arterial compliance, systemic vascular resistance, and effective arterial elastance during exercise in endurance-trained men. Am J Physiol Regul Integr Comp Physiol. 2008; 295(1):R228-35.

17 - Monge García MI, Saludes Orduña P, Cecconi M. Understanding arterial load. Intensive Care Med. 2016; 42(10):1625-27. 
18 - Chemla D, Antony I, Lecarpentier Y, et al. Contribution of systemic vascular resistance and total arterial compliance to effective arterial elastance in humans. Am J Physiol Heart Circ Physiol. 2003; 285(2):H614-20.

19 - Savoia C, Battistoni A, Calvez V, et al. Microvascular Alterations in Hypertension and Vascular Aging. Curr Hypertens Rev. 2017;13(1):16-23.

20 - Sorop O, Olver TD, van de Wouw J, et al. The microcirculation: a key player in obesity-associated cardiovascular disease. Cardiovasc Res. 2017; 113(9):1035-45.

21 - McEvoy JW, Chen Y, Rawlings A, et al. Diastolic blood pressure, subclinical myocardial damage, and cardiac events: implications for blood pressure control. J Am Coll Cardiol. 2016;68:1713-22.

22 - Polese A, De Cesare N, Montorsi P, et al. Upward shift of the lower range of coronary flow autoregulation in hypertensive patients with hypertrophy of the left ventricle. Circulation. 1991;83:84553.

23 - Schiffrin EL. Vascular stiffening and arterial compliance. Implications for systolic blood pressure. Am J Hypertens. 2004; 17(12 Pt 2):39S-48S.

24 - Srichai MB, Lim RP, Wong S, et al. Cardiovascular applications of phase-contrast MRI. Am J Roentgenol. 2009;192(3):662-75.

25 - Akima T, Takase B, Kosuda S, et al. Systemic peripheral vascular resistance as a determinant of functional cardiac reserve in response to exercise in patients with heart disease. Angiology. 2007;58(4):463-71.

26 - Cole RT, Gheorghiade M, Georgiopoulou VV, et al. Reassessing the use of vasodilators in heart failure. Expert Rev Cardiovasc Ther. 2012;10(9):1141-51.

27 - Gandy SJ, Waugh SA, Nicholas RS, et al. Comparison of the reproducibility of quantitative cardiac left ventricular assessments in healthy volunteers using different MRI scanners: a multicenter simulation. J Magn Reson Imaging. 2008;28(2):359-65.

\section{Figures}




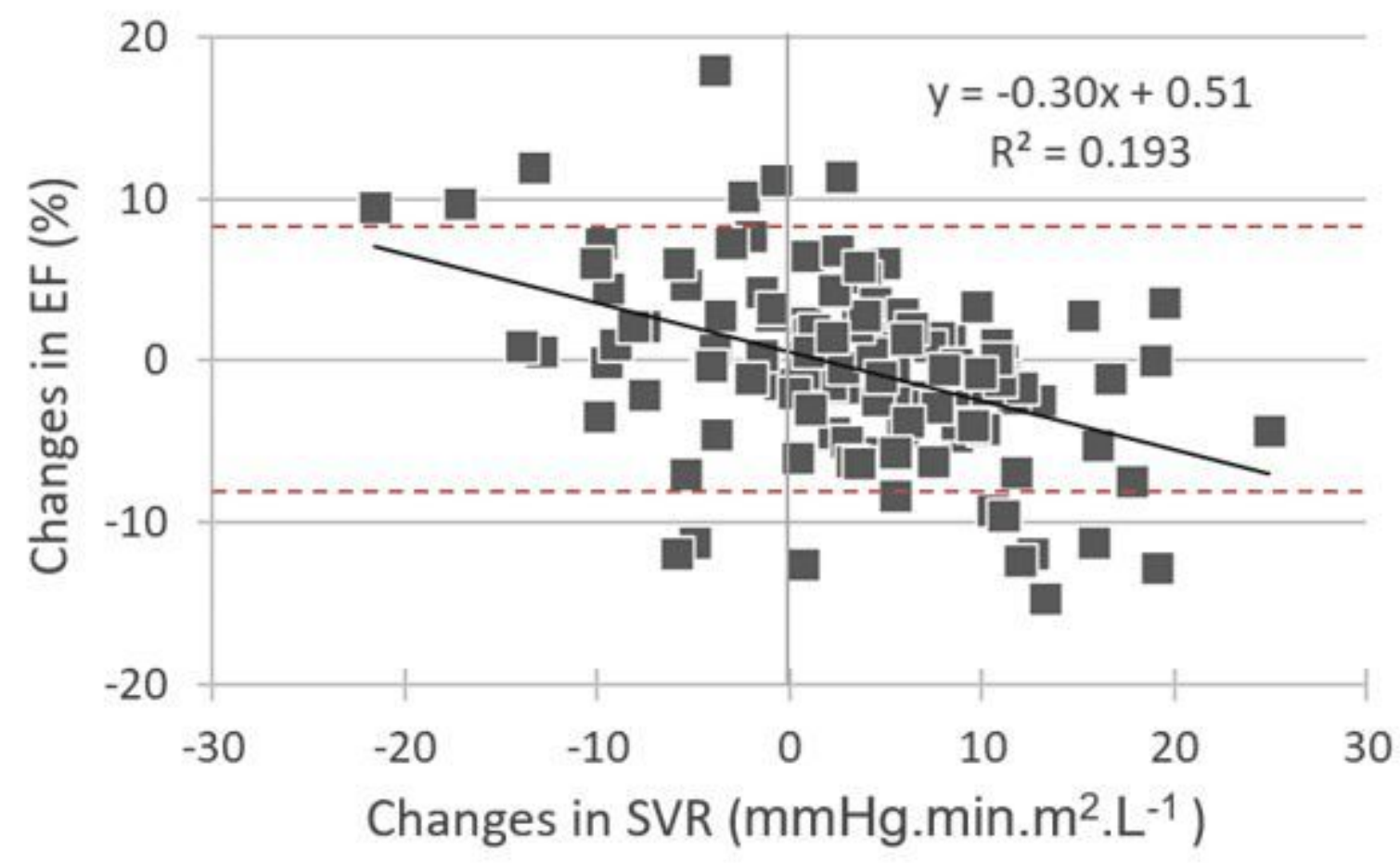

Figure 1

Correlations between baseline-to-follow-up differences in: (i) left ventricular ejection fraction (EF changes) and (ii) systemic vascular resistance (SVR changes) (upper panel). It may additionally be observed that many EF changes are outside of the $-8 \%$ to $+8 \%$ interval (red dashed lines) and may thus be considered significant. 


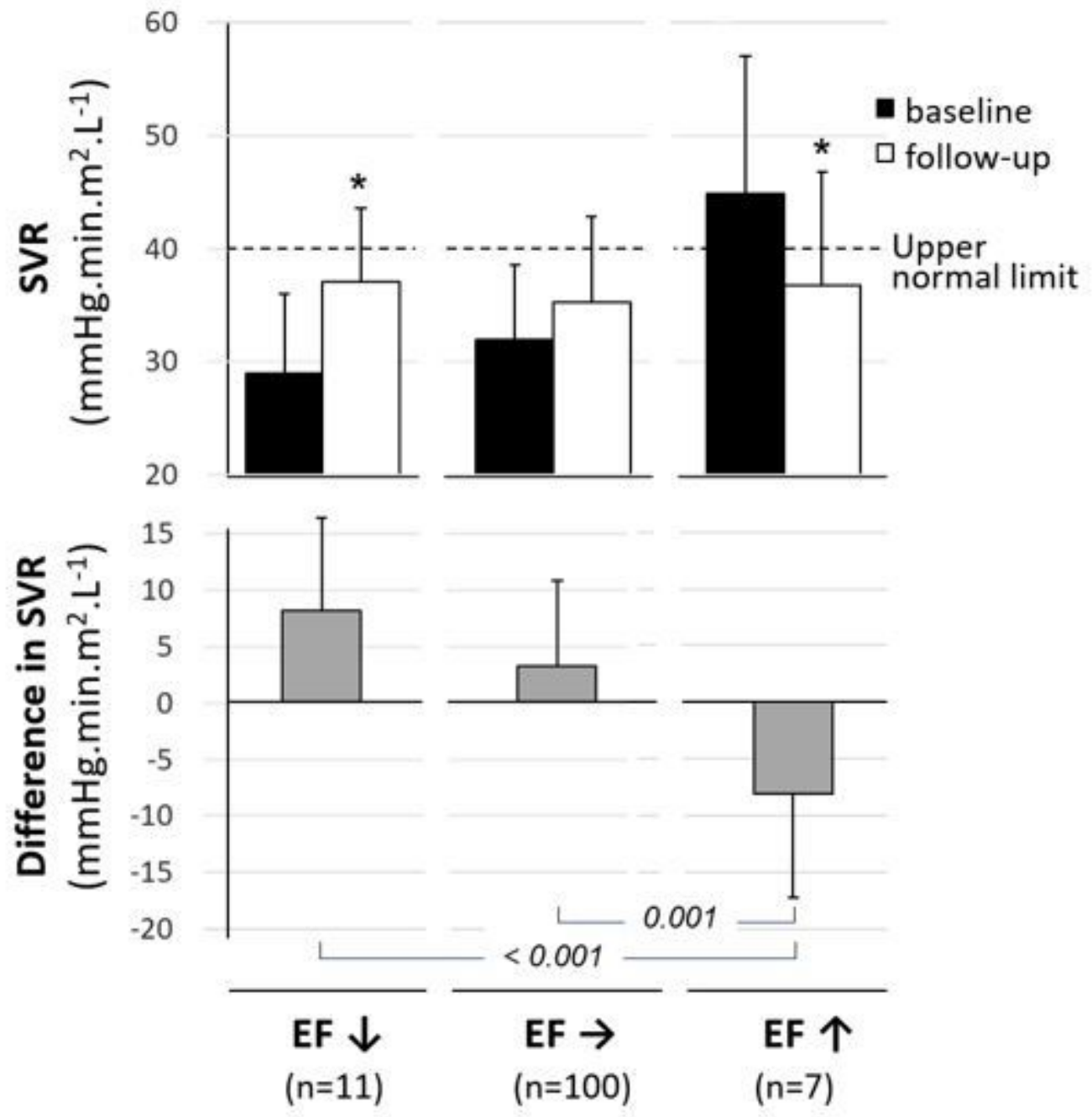

Figure 2

Mean values ( $\pm S D$ ) for baseline (black columns) and follow-up (white columns) levels of systemic vascular resistance (SVR, upper panel) and for the mean difference in SVR between baseline and followup (grey columns, median panel) in subjects categorized in 3 groups based on their baseline-to-follow-up variations in LV ejection fraction -i.e. significant decrease (EF $\downarrow$ ), significant increase (EF $\uparrow)$ and stable $E F$ $(E F \rightarrow)$ *: $p<0.05$ for paired comparisons between baseline and 6 months. 


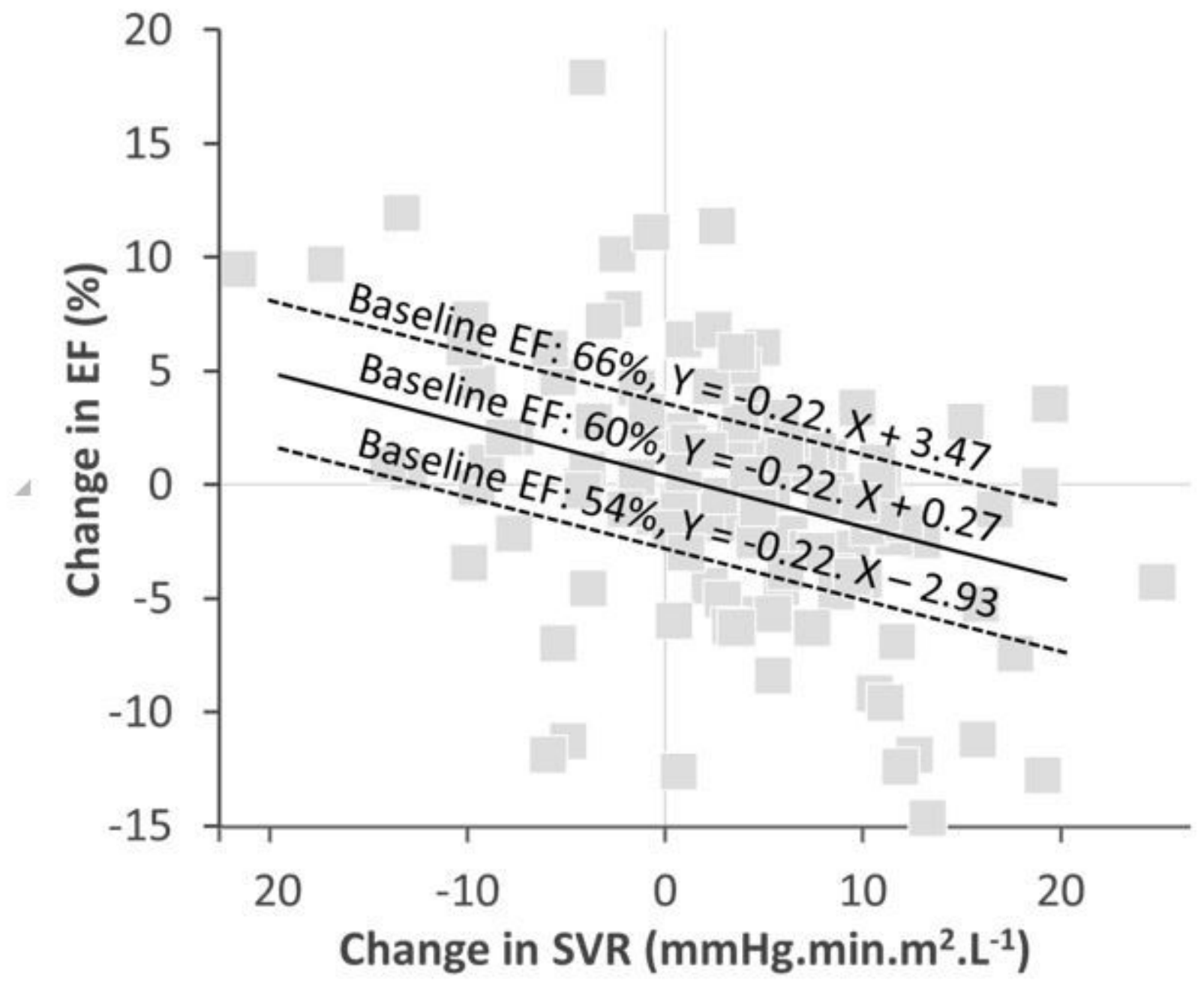

Figure 3

Graph of the correlation between EF-changes and SVR-changes with the regressions computed with the equation from Table 3 for three baseline EF levels: close to the mean (60\%), one SD above the mean (66\%), and one SD below the mean (54\%). The slope of EF- and SVR-changes for the 3 baseline FE levels are identical. However, the intercept corresponding to an absence of any SVR variation, are different, with a predicted absolute decrease in EF of approximately 3\% for the $66 \% \mathrm{EF}$ baseline, an increase of about $3 \%$ for the $54 \%$ EF baseline and an insignificant predicted change for the $60 \%$ EF baseline. 